\title{
Modified Tellegen Principle Used for Power and Energy Systems Modeling
}

\author{
Milan Stork \\ Department of Applied Electronics and \\ Telecommunications/RICE \\ University of West Bohemia \\ Plzen, Czech Republic \\ stork@kae.zcu.cz
}

\author{
Daniel Mayer \\ Department of Theoretical Electrical \\ Engineering \\ University of West Bohemia \\ Plzen, Czech Republic \\ mayer@kte.zcu.cz
}

\begin{abstract}
The paper deals with a problem of modeling of dynamic systems. The proposed approach to the problem solution is based on modified Tellegen's theorem well known from electrical engineering. The novelty of this approach is that it is based on the instantaneous power calculation for real linear and nonlinear systems e.g. electrical circuits, mechanical systems, heat transfer etc. Consequently, mathematically as well as physically correct results are obtained. Some known and often used system representation structures are discussed from the developed point of view as an addition. The examples are also included. The mathematical derivation and results of simulations are presented in this paper.
\end{abstract}

Keywords - dissipation; energy; power; scalar product; state space; Tellegen

\section{INTRODUCTION}

It is familiar that there are two basic approaches to system modeling. The first one consists in using mathematical formulas and physical tools (a causality principle, different forms of conservation laws, power balance relations, etc.) in order to describe appropriate system behavior. It has successfully been used in many fields of science and engineering so far. However, there are also situations where physical laws are not known or cannot be expressed in a proper mathematically exact form. In that case the second basic approach to system modeling can be used. It is based on identification methods working in terms of experimentally gained data. The main aim of this contribution is to formulate power and energetic approach which can be used for different types of system. The method starts from the assumption that any physically correct system representation holds power conservation principle. Such approach can be used for different type of systems.

\section{Modified TELLEGEN PRINCIPLE}

Let $N$ be a physically correct electrical circuit with

Milan Stork's participation was supported by Department of Applied Electronics and Telecommunications, University of West Bohemia, Plzen, Czech Republic and by the Ministry of Education, Youth and Sports of the Czech Republic under the RICE - New Technologies and Concepts for Smart Industrial Systems, project No. LO1607 and the Internal Grant Agency of University of West Bohemia in Plzen, the project SGS-2018-001 the $k$ lumped parameters. Parameters of $N$ can be linear or nonlinear, hysteresis or non-hysteresis, timeconstant or variable. The currents in the branches are $i_{k}(t)$ and the voltage on these branches are $v_{\mathrm{k}}()$. Theorem 1. (Classic Tellegen's theorem [1]). For branch currents $i_{k}(t)$ and branch voltages $v_{k}(t)$ holds true:

$$
\left\langle i(t)^{T}, v(t)\right\rangle=\sum_{k=1}^{b} i_{k}(t) v_{k}(t)=0
$$

It is worth noticing a close relation between first and second Kirchhoff's laws (ensuring physical correctness of $N$ ) and Tellegen's theorem. It is also important to note that inner product according (1) include instantaneous power dissipated on resistors and instantaneous power on inductors and capacitors. Finally, let's note that Tellegen's theorem applies not only to electrical circuits but to any model of a physical correct system with lumped parameters, for example mechanical, thermal, etc [2 - 4]. Therefore if the system is described appropriately by state space equations, voltages and currents are substituted by state space variables and it's derivations.

$$
\left\langle x(t)^{T}, \dot{x}(t)\right\rangle=x_{1}(t) \dot{x}_{1}(t)+x_{2}(t) \dot{x}_{2}(t) \ldots=0
$$

It is worth noticing a close relation between physical correctness and Tellegen's theorem. It is possible describe power or energy in some system by means of Tellegen's theorem. It is shown in first simple example.

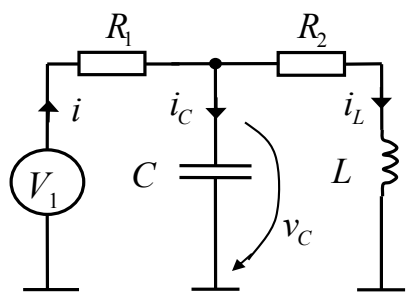

Figure 1. Simple circuit used for example 1

Example1: The state space equations of Fig. 1 are 


$$
\begin{aligned}
& L \frac{d i_{L}}{d t}=-R_{2} i_{L}+v_{C} \\
& C \frac{d v_{C}}{d t}=-i_{L}-\frac{1}{R_{1}} v_{C}+\frac{V_{1}}{R_{1}}
\end{aligned}
$$

The system according by eq. (3) can be described in state space as

$$
\dot{x}(t)=A(t) x(t)+B(t) u(t) ; y(t)=C(t) x(t)
$$

therefore

$$
\begin{aligned}
& \frac{d x_{1}}{d t}=-\frac{R_{2}}{L} x_{1}+\frac{1}{L} x_{2} \\
& \frac{d x_{2}}{d t}=-\frac{1}{C} x_{1}-\frac{1}{C R_{1}} x_{2}+\frac{V_{1}}{C R_{1}}
\end{aligned}
$$

in matrix forms

$$
\left[\begin{array}{c}
\frac{d x_{1}}{d t} \\
\frac{d x_{2}}{d t}
\end{array}\right]=\underbrace{\left[\begin{array}{cc}
-\frac{R_{2}}{L} & \frac{1}{L} \\
-\frac{1}{C} & -\frac{1}{C R_{1}}
\end{array}\right]}_{A} \cdot\left[\begin{array}{l}
x_{1} \\
x_{2}
\end{array}\right]+\underbrace{\left[\begin{array}{c}
0 \\
\frac{1}{C R_{1}}
\end{array}\right]}_{B} \cdot V_{1}
$$

The system according (6) can be transformed by state-space similarity transformation

$$
A_{1}=T \cdot A \cdot T^{-1} ; B_{1}=T \cdot B ; C_{1}=C \cdot T^{-1}
$$

The transformation matrices are

$$
T=\left[\begin{array}{cc}
\sqrt{L} & 0 \\
0 & \sqrt{C}
\end{array}\right] ; \quad T^{-1}=\left[\begin{array}{cc}
1 / \sqrt{L} & 0 \\
0 & 1 / \sqrt{C}
\end{array}\right]
$$

Matrices $A$ and $B$ after transformation (marked as $A_{1}$ and $B_{1}$ ) creates generalized system

$$
A_{1}=\left[\begin{array}{cc}
-\alpha_{11} & \alpha_{2} \\
-\alpha_{2} & -\alpha_{22}
\end{array}\right] ; \quad B_{1}=\left[\begin{array}{c}
0 \\
\beta_{2}
\end{array}\right]
$$

where

$$
\alpha_{11}=\frac{R_{2}}{L} ; \alpha_{2}=\frac{1}{\sqrt{C L}} ; \alpha_{22}=\frac{1}{C R_{1}} ; \beta_{2}=\frac{1}{\sqrt{C} R_{1}}
$$

where relations between $x_{1} \Leftrightarrow i_{\mathrm{L}}$ and $x_{2} \Leftrightarrow v_{\mathrm{C}}$ are

$$
\bar{x}_{1}=\sqrt{L} \cdot i_{L} ; \bar{x}_{2}=\sqrt{C} \cdot v_{C}
$$

Power is given as inner product

$$
\begin{aligned}
& P(t)=\left\langle\bar{x}(t)^{T}, \frac{d \bar{x}(t)}{d t}\right\rangle=\left[\begin{array}{ll}
\bar{x}_{1} & \bar{x}_{2}
\end{array}\right]\left(A_{1}\left[\begin{array}{l}
\bar{x}_{1} \\
\bar{x}_{2}
\end{array}\right]+B_{1} V_{1}\right) \\
& =-\alpha_{11} \bar{x}_{1}^{2}+\underbrace{\alpha_{2} \bar{x}_{1} \bar{x}_{2}-\alpha_{2} \bar{x}_{1} \bar{x}_{2}}_{0}-\alpha_{22} \bar{x}_{2}^{2}+\beta_{2} V_{1} \cdot \bar{x}_{2}
\end{aligned}
$$

After substitution $P(t)$ is

$$
P(t)=-\frac{R_{2}}{L} \bar{x}_{1}^{2}+\underbrace{\frac{\bar{x}_{1} \bar{x}_{2}}{\sqrt{L C}}-\frac{\bar{x}_{1} \bar{x}_{2}}{\sqrt{L C}}}_{0}-\frac{\bar{x}_{2}^{2}}{C R_{1}}+\frac{V_{1} \cdot \bar{x}_{2}}{\sqrt{C} \cdot R_{1}}
$$

Using (11) and (13) for powers evaluation

$$
\begin{aligned}
& P(t)=-\frac{R_{2}}{L} \bar{x}_{1}^{2}-\frac{\bar{x}_{2}^{2}}{C R_{1}}+\frac{V_{1} \cdot \bar{x}_{2}}{\sqrt{C} \cdot R_{1}} \\
& =-\frac{R_{2}}{L}\left(\sqrt{L} \cdot i_{L}\right)^{2}-\frac{\left(\sqrt{C} \cdot v_{C}\right)^{2}}{C R_{1}}+\frac{V_{1} \cdot\left(\sqrt{C} \cdot v_{C}\right)}{\sqrt{C} \cdot R_{1}}
\end{aligned}
$$

After some manipulations

$$
P(t)=\underbrace{V_{1} \frac{V_{1}-v_{C}}{R_{1}}}_{P_{i}} \underbrace{-R_{2} i_{L}^{2}-\frac{\left(V_{1}-v_{C}\right)^{2}}{R_{1}}}_{P_{D}}=0
$$

The power $P(t)$ described by (15) consists from input power $P_{i}$ and two dissipations powers $P_{D}$ (dissipation on $R_{1}$ and $R_{2}$ ).

The real RLC system (see Fig. 1) after similarity transformation is artificial. Moreover the transformation is complicated for high order systems or nonlinear systems. Therefore the new approach (generalized and modified Tellegen's theorem) based was derived. The main difference is that matrix $Q$ contains of energy storage elements is used

$$
P_{M}(t)=\left\langle(Q \cdot x(t))^{T}, \frac{d x(t)}{d t}\right\rangle
$$

where $P_{M}(t)$ is dissipated power. The matrix $Q$ is square diagonal matrix size of $n$. For example 1, the matrix $Q$ is

$$
Q=\left[\begin{array}{ll}
L & 0 \\
0 & C
\end{array}\right]
$$

Therefore $P_{M}(t)$ is

$$
\begin{aligned}
& P_{M}(t)=\left\langle(Q \cdot x(t))^{T}, \frac{d x(t)}{d t}\right\rangle= \\
& \left\langle\left(\left[\begin{array}{cc}
L & 0 \\
0 & C
\end{array}\right] \cdot\left[\begin{array}{c}
x_{1} \\
x_{2}
\end{array}\right]\right)^{T},\left[\begin{array}{c}
\dot{x}_{1} \\
\dot{x}_{2}
\end{array}\right]\right\rangle= \\
& \left.\left\langle\begin{array}{ll}
L x_{1} & C x_{2}
\end{array}\right],\left[\begin{array}{l}
-\frac{R_{2}}{L} x_{1}+\frac{1}{L} x_{2} \\
-\frac{1}{C} x_{1}-\frac{1}{C R_{1}} x_{2}+\frac{V_{1}}{C R_{1}}
\end{array}\right]\right\rangle
\end{aligned}
$$

result of the scalar product is

$$
\begin{aligned}
& P_{M}(t)=\left\langle Q x(t), \frac{d x(t)}{d t}\right\rangle= \\
& -R_{2} x_{1}^{2}+\underbrace{x_{1} x_{2}-x_{1} x_{2}}_{0}-\frac{1}{R_{1}} x_{2}^{2}+\frac{V_{1}}{R_{1}} x_{2}
\end{aligned}
$$


where $x_{I}=i_{L}$ and $x_{2}=v_{C}$ and therefore result is the same as eq. (15) without unpleasant transformation. For system modelling it is possible use 2 types of models: M1-RLC for systems with complex conjugate poles and also for systems with only real poles, M2 for systems with real poles (RC or RL models), circuit diagram of the models are shown in Fig. 2, 3 and 4.

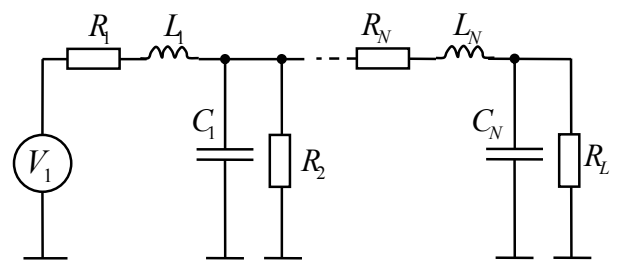

Figure 2. The ladder structure of RLC model with complex conjugate and also real poles. Model M1-RLC

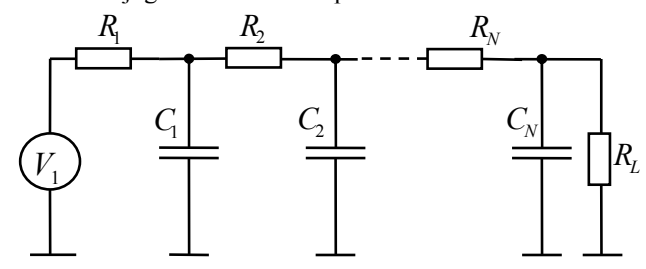

Figure 3. The ladder structure of RC model with real poles. Model M2-RC

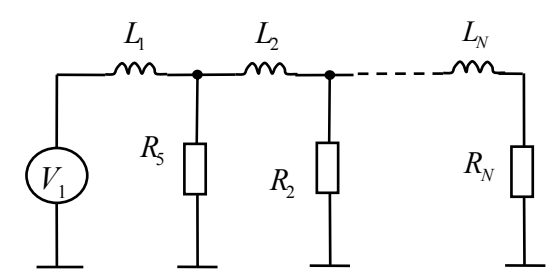

Figure 4. The ladder structure of RL model with real poles. Model M2-RL

The state space equation for model M1-RLC is eq. (20), for model M2-RC it is eq. (29) - only matrix $A$ is presented. Eq. for model M2-RL is similar (not shown here). Equations are for $4^{\text {th }}$ orders models only.

$$
\left[\begin{array}{c}
\frac{d i_{L 1}}{d t} \\
\frac{d u_{C 1}}{d t} \\
\frac{d i_{L 2}}{d t} \\
\frac{d u_{C 2}}{d t}
\end{array}\right]=\left[\begin{array}{cccc}
-\frac{R_{1}}{L_{1}} & -\frac{1}{L_{1}} & 0 & 0 \\
\frac{1}{C_{1}} & -\frac{1}{C_{1} R_{2}} & -\frac{1}{C_{1}} & 0 \\
0 & \frac{1}{L_{2}} & -\frac{R_{3}}{L_{2}} & -\frac{1}{L_{2}} \\
0 & 0 & \frac{1}{C_{2}} & -\frac{1}{C_{2} R_{4}}
\end{array}\right]\left[\begin{array}{c}
i_{L 1} \\
u_{C 1} \\
i_{L 2} \\
u_{C 2}
\end{array}\right]+\left[\begin{array}{c}
\frac{1}{L_{1}} \\
0 \\
0 \\
0
\end{array}\right] V_{1}
$$

with property (for linear systems)

$$
\sum_{j=1}^{n} a_{i j}=0 ; \quad i=2,3 \ldots n-1 ; \quad i \neq j
$$

where matrix $Q$ for M1-RLC - i.e. eq. (20) is

$$
Q=\left[\begin{array}{cccc}
L_{1} & 0 & 0 & 0 \\
0 & C_{1} & 0 & 0 \\
0 & 0 & L_{2} & 0 \\
0 & 0 & 0 & C_{2}
\end{array}\right]
$$

Power $P_{M}$ for M1-RLC is calculated as

$$
\begin{aligned}
& P_{M}=\left\langle(Q x)^{T}, \frac{d x}{d t}\right\rangle=L_{1} x_{1}\left(-\frac{R_{1} x_{1}}{L_{1}}-\frac{x_{2}}{L_{1}}+\frac{V_{1}}{L_{1}}\right)+ \\
& C_{1} x_{2}\left(\frac{x_{1}}{C_{1}}-\frac{x_{2}}{C_{1} R_{2}}-\frac{x_{3}}{C_{1}}\right)+L_{2} x_{3}\left(\frac{x_{2}}{L_{2}}-\frac{R_{3} x_{3}}{L_{2}}+\frac{x_{4}}{L_{2}}\right)+ \\
& C_{2} x_{4}\left(\frac{x_{3}}{C_{2}}-\frac{x_{4}}{C_{2} R_{4}}\right)
\end{aligned}
$$

and result after some manipulation is

$$
\begin{aligned}
& P_{M}=-R_{1} x_{1}^{2}+V_{1} x_{1}-\frac{x_{2}^{2}}{R_{2}}-R_{3} x_{3}^{2}-\frac{x_{4}^{2}}{R_{4}} \\
& \underbrace{V_{1} x_{1}}_{P i} \underbrace{-R_{1} x_{1}^{2}-\frac{x_{2}^{2}}{R_{2}}-R_{3} x_{3}^{2}-\frac{x_{4}^{2}}{R_{4}}}_{P d}
\end{aligned}
$$

Example2: Differential equation which represents some dynamical system is

$$
x^{(4)}+x^{(3)}+3 x^{(2)}+0.8 \dot{x}+x=0
$$

From eq. (20) and generalized eq. (26) (M1-RLC) it is possible find many solutions of eq. (25) in state space representations. The energy storage elements are 4 and dissipation elements can be from 1 to 4 .

$\left[\begin{array}{l}\frac{d x_{1}}{d t} \\ \frac{d x_{2}}{d t} \\ \frac{d x_{3}}{d t} \\ \frac{d x_{4}}{d t}\end{array}\right]=\left[\begin{array}{cccc}-\frac{d_{1}}{q_{1}} & -\frac{1}{q_{1}} & 0 & 0 \\ \frac{1}{q_{2}} & -\frac{d_{2}}{q_{2}} & -\frac{1}{q_{2}} & 0 \\ 0 & \frac{1}{q_{3}} & -\frac{d_{3}}{q_{3}} & -\frac{1}{q_{3}} \\ 0 & 0 & \frac{1}{q_{4}} & -\frac{d_{4}}{q_{4}}\end{array}\right]\left[\begin{array}{l}x_{1} \\ x_{2} \\ x_{3} \\ x_{4}\end{array}\right]+\left[\begin{array}{c}\frac{1}{q_{1}} \\ 0 \\ 0 \\ 0\end{array}\right] V_{1}$

$d_{i}-$ dissipation, $q_{i}$ - energy storage element. One of many possible solutions is (matrix has the same eigenvalues): $\quad d_{1}=1 ; \quad d_{2}=0 ; \quad d_{3}=0 ; \quad d_{4}=0 ; \quad q_{1}=1$; $q_{2}=0.4545 ; q_{3}=6.37 ; q_{4}=0.346$. The system with one dissipation is given in eq. (27)

$$
\left[\begin{array}{l}
\dot{x}_{1} \\
\dot{x}_{2} \\
\dot{x}_{3} \\
\dot{x}_{4}
\end{array}\right]=\left[\begin{array}{cccc}
-1 & -1 & 0 & 0 \\
2.2 & 0 & -2.2 & 0 \\
0 & 0.157 & 0 & -0.157 \\
0 & 0 & 2.89 & 0
\end{array}\right]\left[\begin{array}{l}
x_{1} \\
x_{2} \\
x_{3} \\
x_{4}
\end{array}\right]+\left[\begin{array}{l}
1 \\
0 \\
0 \\
0
\end{array}\right] V_{1}
$$

or $C_{2}=$ parameter and $L_{l}=1 /\left(2.89 \cdot C_{2}\right) ; \quad C_{l}=C_{2} / 0.76$; $L_{2}=1 /\left(0.4545^{*} C_{2}\right) ; R_{1}=L_{1}$; see eq. (19). Other solution, i.e. system with 2 dissipations is 


$$
\left[\begin{array}{c}
\dot{x}_{1} \\
\dot{x}_{2} \\
\dot{x}_{3} \\
\dot{x}_{4}
\end{array}\right]=\left[\begin{array}{cccc}
-0.85 & -2.35 & 0 & 0 \\
1 & 0 & -1 & 0 \\
0 & 0.08 & 0 & -0.08 \\
0 & 0 & 5.13 & -0.154
\end{array}\right]\left[\begin{array}{l}
x_{1} \\
x_{2} \\
x_{3} \\
x_{4}
\end{array}\right]+\left[\begin{array}{c}
2.35 \\
0 \\
0 \\
0
\end{array}\right] V_{1}
$$

The systems according eq. (27) and (28) (M1RLC) are real systems with $R L C$ components, e.g. for eq. (28) and according eq. (20): $R_{I}=0.362 ; L_{l}=0.43$; $C_{1}=1 ; L_{2}=12.5 ; C_{2}=0.195 ; R_{4}=33.3$.

For M2-RC (only matrix $A$ is presented) matrix is

$$
A=\left[\begin{array}{cccc}
\frac{-1}{C_{1}}\left(\frac{1}{R_{1}}+\frac{1}{R_{2}}\right) & \frac{1}{C_{1} R_{2}} & 0 & 0 \\
\frac{1}{C_{2} R_{2}} & \frac{-1}{C_{2}}\left(\frac{1}{R_{2}}+\frac{1}{R_{3}}\right) & \frac{1}{C_{2} R_{3}} & 0 \\
0 & \frac{1}{C_{3} R_{3}} & \frac{-1}{C_{3}}\left(\frac{1}{R_{3}}+\frac{1}{R_{4}}\right) & \frac{1}{C_{3} R_{4}} \\
0 & 0 & \frac{1}{C_{4} R_{4}} & \frac{-1}{C_{4}}\left(\frac{1}{R_{4}}+\frac{1}{R_{5}}\right)
\end{array}\right]
$$

with property (for linear systems)

$$
\sum_{j=1}^{n} a_{i j}=0 ; \quad i=2,3 \ldots n-1
$$

Example 3: Differential equation for M2-RC is given in eq. (31)

$$
x^{(4)}+10 x^{(3)}+35 x^{(2)}+50 \dot{x}+24 x=0
$$

Equation (31) can be converted to system

$$
\left[\begin{array}{l}
\dot{x}_{1} \\
\dot{x}_{2} \\
\dot{x}_{3} \\
\dot{x}_{4}
\end{array}\right]=\left[\begin{array}{cccc}
-2.82 & 0.62 & 0 & 0 \\
1.84 & -2.16 & 0.32 & 0 \\
0 & 2.73 & -2.85 & 0.12 \\
0 & 0 & 2.17 & -2.17
\end{array}\right]\left[\begin{array}{c}
x_{1} \\
x_{2} \\
x_{3} \\
x_{4}
\end{array}\right]+\left[\begin{array}{c}
0.62 \\
0 \\
0 \\
0
\end{array}\right] \cdot V_{1}
$$

It is possible calculate values of resistors and capacitors from (29) and (32) for chosen $C_{1}=1$ (as parameter). Other values are: $R_{1}=0.45 ; C_{2}=0.33$; $R_{2}=1.62 ; C_{3}=0.039 ; R_{3}=9.29 ; C_{4}=0.0022 ; R_{4}=211$ and matrix $Q$ for $\mathrm{M} 2-\mathrm{RC}$ is

$$
Q=\left[\begin{array}{cccc}
C_{1} & 0 & 0 & 0 \\
0 & C_{2} & 0 & 0 \\
0 & 0 & C_{3} & 0 \\
0 & 0 & 0 & C_{4}
\end{array}\right]=\left[\begin{array}{cccc}
1 & 0 & 0 & 0 \\
0 & 0.33 & 0 & 0 \\
0 & 0 & 0.039 & 0 \\
0 & 0 & 0 & 0.0022
\end{array}\right]
$$

therefore according M2-RC (28), (32) and (15) $P_{M}$ is

$$
\begin{aligned}
& P_{M}=\left\langle(Q x)^{T}, \frac{d x}{d t}\right\rangle=C_{1} \cdot x_{1} \cdot \frac{d x_{1}}{d t}+ \\
& C_{2} \cdot x_{2} \cdot \frac{d x_{2}}{d t}+C_{3} \cdot x_{3} \cdot \frac{d x_{3}}{d t}+C_{4} \cdot x_{4} \cdot \frac{d x_{4}}{d t}
\end{aligned}
$$

after adjustments (34) according to the equation (23)

$$
\begin{aligned}
P_{M}= & V_{1} \frac{V_{1}-v_{C 1}}{R_{1}}-\frac{\left(V_{1}-v_{C 1}\right)^{2}}{R_{1}}-\frac{\left(v_{C 1}-v_{C 2}\right)^{2}}{R_{2}} \\
& -\frac{\left(v_{C 2}-v_{C 3}\right)^{2}}{R_{3}}-\frac{\left(v_{C 3}-v_{C 4}\right)^{2}}{R_{4}}
\end{aligned}
$$

Energy of the system is calculated according

$$
E(t)=\int_{0}^{\infty} P_{M}(t) d t
$$

where $q_{i i}$ are diagonal elements of $Q$.

Example 4: Nonlinear autonomous dissipative Duffing system differential equation is

$$
\ddot{x}+b \dot{x}-x+x^{3}=0
$$

where dissipative parameter $b=0.1$. In state space

$$
\begin{aligned}
& \dot{x}=y \\
& \dot{y}=-b y+x-x^{3}
\end{aligned}
$$

Previous equations can be rewritten as nonlinear electrical system

$$
\begin{aligned}
& \frac{d v_{C}}{d t}=\frac{1}{C} i_{L} \\
& \frac{d i_{L}}{d t}=\frac{1}{L}\left(-R \cdot i_{L}+v_{C}-v_{C}^{3}\right)
\end{aligned}
$$

Power $P_{M}$ given by scalar product is

$$
\begin{aligned}
P_{M} & =\left\langle\left(\left[\begin{array}{cc}
C & 0 \\
0 & L
\end{array}\right] \cdot\left[\begin{array}{c}
v_{C} \\
i_{L}
\end{array}\right]\right)^{T},\left[\begin{array}{c}
\dot{v}_{C} \\
i_{L}
\end{array}\right]\right\rangle \\
& =v_{C} \cdot i_{L}+i_{L} \cdot v_{C}-i_{L} \cdot v_{C}^{3}-R \cdot i_{L}^{2}
\end{aligned}
$$

The simulation results are shown in Fig. 5 to Fig. 6 which presents time evolution of signals, Fig. 7 and 8 concerning power $P_{M}$ and only dissipated power $-R \cdot i_{L}^{2}$, Fig, 9 and 10 which displays energy of the system and dissipated energy respective. It should be noticed that energy is calculated by integration of power.

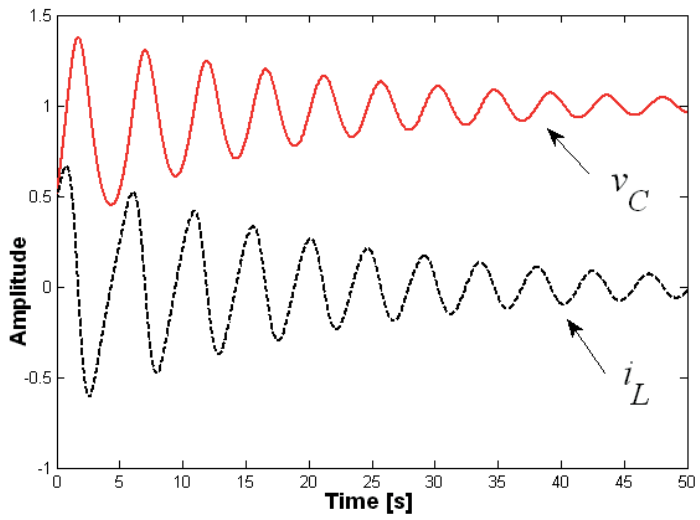

Figure 5. Dissipative Duffing system. Time evolution of $v_{C}$ and $i_{L}$ signals 


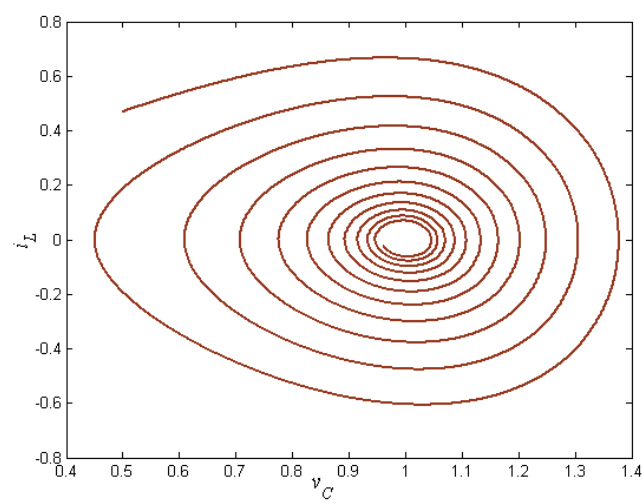

Figure 6. Dissipative Duffing system. Phase space evolution of $v_{C}$ and $i_{L}$ signals

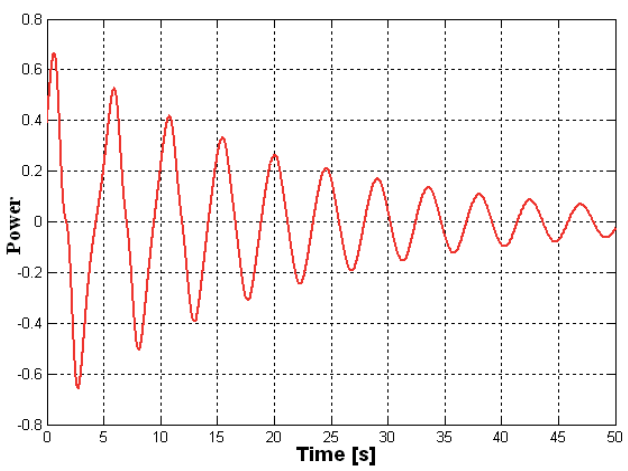

Figure 7. Dissipative Duffing system. Time evolution of power $P_{M}$ according (40)

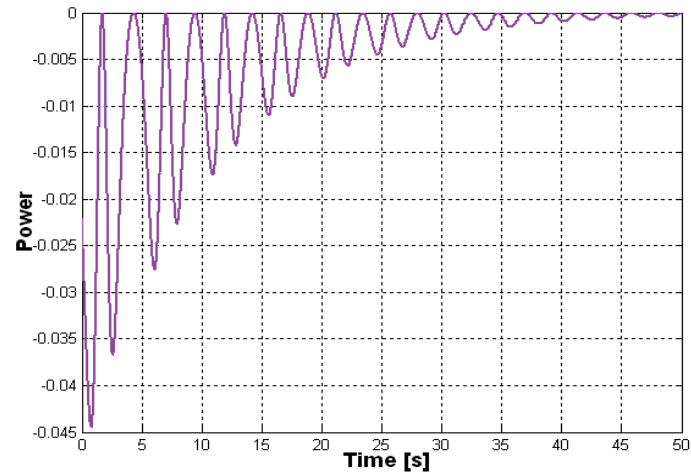

Figure 8. Dissipative Duffing system. Time evolution of dissipative power given by $-R \cdot i_{L}^{2}$

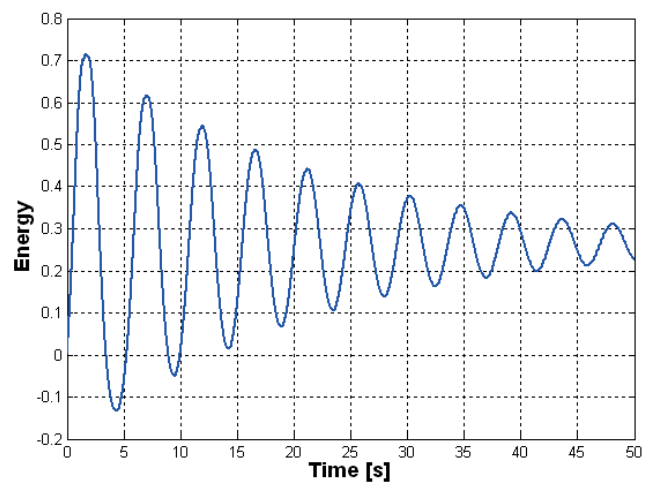

Figure 9. Dissipative Duffing system. Time evolution of energy given by integration of $P_{M}$

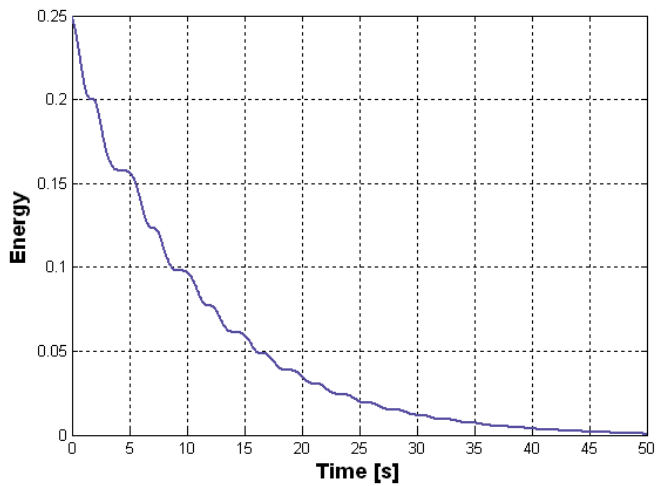

Figure 10. Dissipative Duffing system. Time evolution of dissipative energy given by integration $-R \cdot i_{L}^{2}$

\section{CONCLUSION}

In this paper the power and energy approach based on generalized Tellegen principle was used for linear and nonlinear dynamical systems. The theory was confirmed by simulation on several examples. Presented practice can be used for different types of systems which can be described by ordinary differential equation or set of differential equations.

\section{REFERENCES}

[1] J. Hrusak, D. Mayer, M. Stork, "On System Structure Reconstruction Problem And Tellegen-Like Relations", Proc. of $8^{\text {th }}$ World Multiconf., SCI, Vol. VIII, Florida, USA, 2004, pp. 373-378

[2] Y. W. Yan and K. L. Chung, A Fast Algorithm for Solving Special Tridiagonal Systems, Computing, 1994, vol 52, pp. 203-211.

[3] D. Mayer, J. Hrusak \& M. Stork, "On state-space energy based generalization of Brayton-Moser topological approach to electrical network decomposition,". Springer, Computing, 2013, DOI 10.1007/s00607-012-0280-2.

[4] A. J. Van der Schaft and B. M. Maschke, "The Hamiltonian Formulation of Energy Conserving Physical Systems with External Ports," Archiv fuer Elektronik und Uebertragungstechnik, 1995, 49 (5/6), pp. 362-371. 